\title{
Au-Silica Core/Shell Hybrid Nanoparticles Furnished with CdTe Nanocrystals for Enhanced Plasmon-Exciton Interactions
}

\author{
Ilkem Ozge Ozel, ${ }^{1}$ Tuncay Ozel, ${ }^{1}$ Donus Tuncel, ${ }^{1}$ and Hilmi Volkan Demir ${ }^{1,2, *}$ \\ ${ }^{1}$ Department of Physics, Department of Chemistry, Department of Electrical and Electronics Engineering, and \\ UNAM - Institute of Materials Science and Nanotechnology, Bilkent University, TR-06800, Ankara, Turkey \\ ${ }^{2}$ Nanyang Technological University, School of Electrical \& Electronic Engineering, Division of Microelectronics, \\ School of Mathematical and Physical Sciences, Luminous! Semiconductor Lighting and Display Center of \\ Excellence, Nanyang Avenue, Singapore 639798, Singapore \\ *Tel:[+90]( 312) 290 1021, e-mail: volkan@bilkent.edu.tr
}

To date extensive research efforts have confirmed that optical properties of bulk semiconductors and metals are substantially modified when they are transformed into nano-dimensions. ${ }^{1}$ For example, metal nanoparticles with dimensions less than hundred nanometers strongly oscillate at a specific resonance frequency to generate localized surface plasmons depending on their size. This creates strongly localized electric fields in the close proximity of the metal nanoparticles under external excitation. Similarly, because of the size effect, semiconductor quantum dot nanocrystals (NCs), with dimensions less than 10 nanometers, feature highly tunable absorption and emission characteristics. High levels of brightness and photostability, and broad excitation range with sharp emission spectra make these NCs ideal candidates as active materials in light emitting diodes and solar cells, and as fluorescent labels in bioimaging. ${ }^{1-4}$ More recently, combining these two sets of nanomaterials has received increasing attention for their possible end uses of light harvesting and light generation in optoelectronics and highly promising sensing and imaging applications in biophotonics. ${ }^{2-4}$ Even though $\mathrm{NCs}$ are highly amenable for such a wide range of aforementioned applications, there are still some performance related issues yet to be overcome. One of such main challenges is to increase the fluorescence quantum efficiency of NCs and other is to make them non-blinking. Plasmon coupling of NCs offers possible means to address these and similar problems. This, however, requires hybrid architectures to intimately integrate these nanomaterials. Although there are straightforward methods of simply blending these nanomaterials and making thin films of their blends, or making bilayers of these nanocrystal and metal nanoparticle films on top of each other, such methods typically suffer from either limited control in spacing metal nanoparticles and semiconductor nanocrystals precisely, or from total film thickness of bilayers that can be put together. In this work, we developed and demonstrated Au-silica core/shell nanoparticles that successfully assemble CdTe nanocrystals right on their silica shells for enhanced plasmon-exciton interactions, while avoiding the problems of lacking control in spacing and limited film thickness. Herein we present our synthesis and characterization results of this new set of CdTe NC decorated, Au-silica core/shell hybrid nanoparticles with silica shell thicknesses controlled and tuned by synthesis (Fig. 1.).
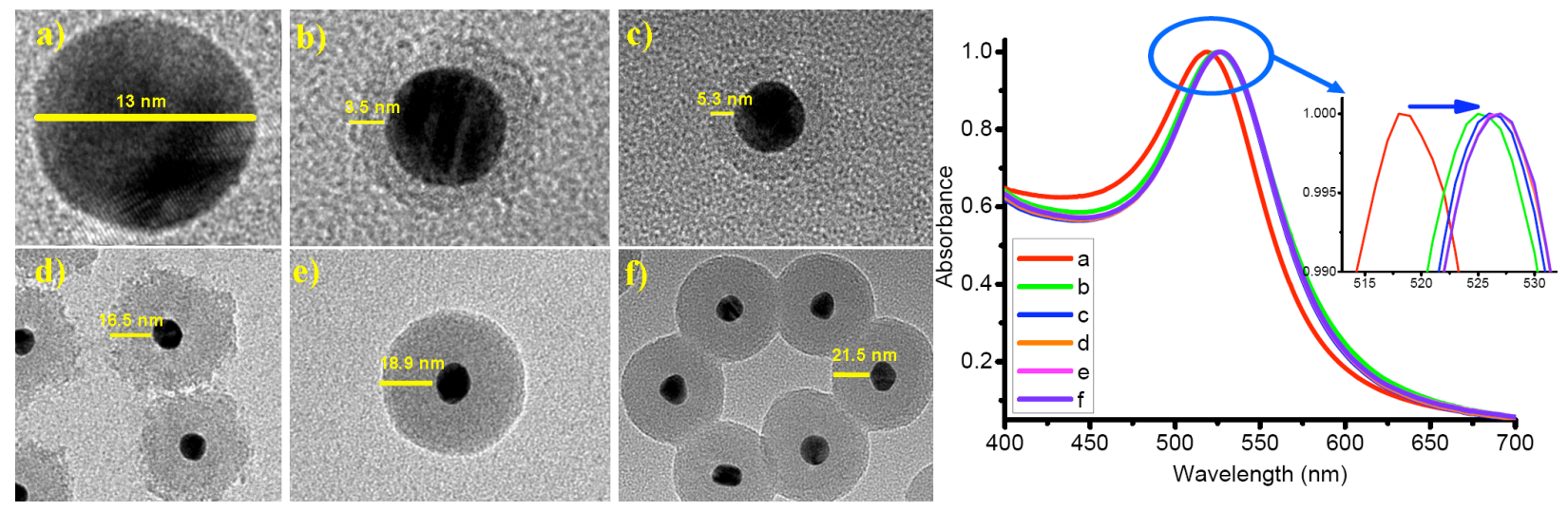

Figure 1. (Left) Transmission electron microscopy (TEM) images of (a) Au nanoparticles alone, and (b)-(f) Au coresilica shell hybrid nanoparticles with silica shell thicknesses controlled and tuned by varying synthesis conditions. (Right) Absorbance spectra of samples given in figures (a)-(f) to monitor shifts in the surface plasmon resonance peaks because of increasing silica thickness. 
In our synthesis procedure, hybrid nanostructures of $\mathrm{Au} / \mathrm{SiO}_{2} / \mathrm{CdTe}-\mathrm{NCs}$ were prepared using a synthetic pathway comprising multiple steps. First, synthesis of gold nanoparticles $\left(5 \times 10^{-4} \mathrm{M}\right)$ was performed according to the standard sodium citrate reduction method, which yields a stable, deep-red gold nanoparticle dispersion with an average diameter of $15 \mathrm{~nm}^{5}$ Further steps involved surface activation of the citrate capped Au nanoparticles with (3Aminopropyl) trimethoxysilane (APS -a silane coupling agent) for $15 \mathrm{~min}$ to achieve complete complexation of amine groups with the gold surface. Next, to complete the silica-shell growth of the silane functionalized Au nanoparticles, a $100 \mathrm{~mL}$ gold sol is allowed to stand for $24 \mathrm{~h}$ under vigorous stirring after addition of a $5 \mathrm{~mL}$ solution of $0.54 \mathrm{wt} \%$ active silica. To facilitate further growth of a thicker silica shell on Au nanoparticles, the Au sol in varying water/ethanol concentrations is mildly stirred for $12 \mathrm{~h}$ after addition of TES (tetraethyl orthosilicate) and ammonia. ${ }^{6}$ In the next step, the silica coated gold nanoparticles are surface treated with APS to form hybrid Ausilica core/shell nanoparticles functionalized with $-\mathrm{NH}_{2}$ groups. Finally, to the sonicated, surface-modified $\mathrm{Au}$ nanoparticle dispersion, a dilute solution of TGA capped CdTe NCs was rapidly added, which results in the aimed hybrid $\mathrm{Au} / \mathrm{SiO}_{2} / \mathrm{CdTe}-\mathrm{NC}$ nano-assembly (Fig. 2). Extensive synthesis studies and TEM characterizations show that it is possible to control and tune the silica shell thickness around Au cores. Furthermore, decorating CdTe NCs on these Au-silica hybrid nanoparticles allows for the intimate integration of NCs with $\mathrm{Au}$ nanoparticles with a precisely controlled spacing shell. Numerical modeling indicates that such a hybrid nano-assembly allows for plasmon-exciton interaction. Time-resolved fluorescence measurements confirm that the decorated nanocrystals are plasmon-coupled and show that their 1/e decay lifetimes are shortened (from $16.7 \mathrm{~ns}$ to $0.7 \mathrm{~ns}$ ) as a result of excitonplasmon interaction.
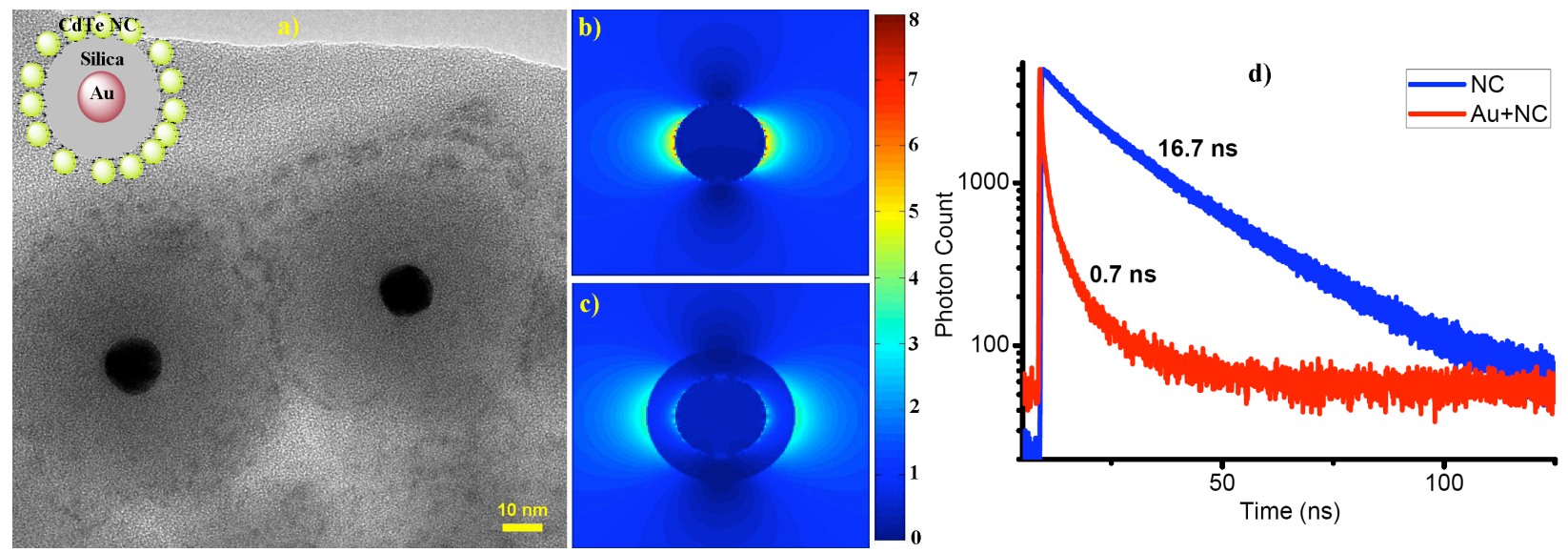

Figure 2. (a) Transmission electron micrograph of hybrid $\mathrm{Au} / \mathrm{SiO}_{2} / \mathrm{CdTe}-\mathrm{NC}$ nano-assembly (onset), schematic representation of this hybrid structure (inset). Electric field intensity maps around (b) a representative Au nanoparticle $(15 \mathrm{~nm})$ and $(\mathrm{c})$ that with a silica shell $(5 \mathrm{~nm})$, showing field localization at their plasmon resonance wavelengths. (d) Time-resolved fluorescence decay curves of CdTe NCs alone and when decorated on Au/silica nanoparticles.

To conclude, we successfully developed and demonstrated the synthesis of varying silica shell thicknesses on gold nanoparticles for plasmon-exciton interactions between self-assembled CdTe NCs and Au core nanoparticles. These hybrid nano-assemblies are highly promising for potential applications ranging from sensing technologies and solidstate lighting to amplification of surface plasmons.

Acknowledgements: This work is supported by NRF-RF, EU-FP7 Nanophotonics4Energy NoE, and TUBITAK EEEAG 107E088, 109E002, 109E004, and 110E010. HVD acknowledges support from ESF-EURYI and TUBAGEBIP.

\section{References}

[1] Gaponenko, S. V. Optical Properties of Semiconductor Nanocrystals; Cambridge University Press: Cambridge, UK, 2005.

[2] Atwater, H. A.; Polman, A. Nat. Mater. 2010, 9, 205-213.

[3] Anker, J. N.; Hall, W. P.; Lyandres, O.; Shah, N. C.; Zhao, J.; Van Duyne, R. P. Nat. Mater. 2008, 7, 442-453.

[4] Gao, X.; Cui, Y.; Levenson, R. M.; Chung, L. W. K.; Nie, S. Nat. Biotechnol. 2004, 22, 969.

[5] Enustun, B. V.; Turkevich, J. J. Am. Chem. Soc. 1963, 85, 3317.

[6] Liz-Marzan, L. M.; Giersig, M.; Mulvaney, P. Langmuir 1996, 12, 4329-4335. 